\title{
Phase behavior and far-from-equilibrium gelation in charged attractive colloids
}

\author{
P. Charbonneau ${ }^{1, *}$ and D. R. Reichman ${ }^{2}$ \\ ${ }^{1}$ Chemistry and Chemical Biology, Harvard University, 12 Oxford Street, Cambridge, Massachusetts 02138, USA \\ ${ }^{2}$ Department of Chemistry, Columbia University, 3000 Broadway, New York, New York 10027, USA
}

(Received 18 April 2006; revised manuscript received 1 March 2007; published 3 May 2007)

\begin{abstract}
In this Rapid Communication we demonstrate the applicability of an augmented Gibbs ensemble Monte Carlo approach for the phase behavior determination of model colloidal systems with short-ranged depletion attraction and long-ranged repulsion. This technique allows for a quantitative determination of the phase boundaries and ground states in such systems. We demonstrate that gelation may occur in systems of this type as the result of arrested microphase separation, even when the equilibrium state of the system is characterized by compact microphase structures.
\end{abstract}

DOI: 10.1103/PhysRevE.75.050401

PACS number(s): 82.70.Dd, 64.60.-i, 61.20.Lc, 61.46.Bc

Understanding the routes to colloidal gel formation is a problem in the forefront of soft condensed matter physics. It has been demonstrated via theory [1], experiment [2,3], and simulation $[4,5]$ that a weak, porous solid may be formed when a suspension of colloidal particles with short-ranged attractions is quenched below its critical point. Spinodal decomposition, expected to lead to complete phase separation into colloid-rich and colloid-poor regions, may arrest or become anomalously slow when bonding between colloidal particles is sufficiently strong. The resulting structure may support weak shear stresses, and is called a colloidal gel. While equilibrium routes to physical colloidal gelation do exist [6-8], the nonequilibrium route described above is perhaps the most ubiquitous.

When excess charge resides on the colloidal particles, the situation is more complex [9]. In addition to short-ranged depletion interactions, the long-ranged repulsive portion of the potential may lead to the formation of various microphase structures, such as clusters, cylinders, sheets, and spirals [10-14]. As a result, the nature of physical gelation in these systems is potentially more subtle than in systems with attractive interactions alone. Indeed, several novel routes to gelation in these systems have been discussed. One proposal is that compact, thermodynamically stable clusters may form the building blocks of a glassy state [15]. At high enough volume fraction clusters may aggregate or become caged, as in the case for individual colloidal particles near the colloidal glass transition [16]. At low volume fractions, such a glassy state could be stabilized by the effective long-ranged repulsion between individual clusters [15]. More recently, it has been proposed that disordered or partially ordered anisotropic domains may be responsible for physical gelation in charged colloidal systems $[11,14,17]$.

A major difficulty in testing the validity of any proposal resides in the accurate calculation of the phase diagram in systems with long-ranged repulsive and short-ranged attractive interactions. When the range of the attractive interaction is comparable to the size of the particles, the phase behavior may be characterized easily with standard simulation tech-

*Current address: FOM Institute for Atomic and Molecular Physics, Kruislaan 407, 1098 SJ Amsterdam, The Netherlands. niques [12-14]. On the other hand, when the attractive portion of the potential varies over distances that are a fraction of the particle size, sampling issues become severe, and direct molecular dynamics (MD) and Monte Carlo (MC) techniques are not feasible. Unfortunately it is in the shortranged attractive limit that the gel phase is usually stabilized $[4,5]$. We have recently demonstrated $[18,19]$ that a judicious implementation of the Gibbs ensemble Monte Carlo (GEMC) [20] technique allows for phase behavior computation in systems with very short-ranged attractive interactions. In this Rapid Communication we adapt the GEMC technique for the study of systems characterized by short-ranged attractions and long-ranged repulsion. The successful application of this approach provides crucial information on possible dynamical routes to gelation in systems with competing interactions, which are examined via MD simulation. Indeed, our conclusions and interpretations of the gelation mechanism differ from those previously drawn from studies of analogous systems.

In this work we study potentials of the form ${ }^{1}$

$$
U(r)=4 \epsilon\left[\left(\frac{\sigma}{r}\right)^{2 n}-\left(\frac{\sigma}{r}\right)^{n}\right]+A \frac{e^{-r / \xi}}{r / \xi},
$$

[15], where large $n$ values correspond to short-ranged attractions. We study three-dimensional systems $(n=50)$ with three sets of repulsive Yukawa parameters $(A=0[18] ; A=1.275$, $\xi=0.5 ; A=0.203, \xi=1)$ and two-dimensional systems ( $n$ =100) with parameters $(A=0 ; A=0.68, \xi=1 ; A=0.2, \xi=2)$. For the last parameter set, the system is beyond the Lifshiftz point and thus microphase separates at low temperatures. A preliminary dynamical study of a related three-dimensional system has been performed by Sciortino et al. [15]. For visualization purposes, we focus on a two-dimensional system. As discussed below we expect all qualitative conclusions to be unmodified by this choice. Selected results will also be provided for the three-dimensional case.

For the vapor-solid equilibrium, we use the GEMC meth-

\footnotetext{
${ }^{1}$ Temperature $T$ is in units of well depth $\epsilon$, distance in units of particle diameter $\sigma$, and time $t$ in units of $\sqrt{\epsilon / m \sigma^{2}}$ with particle mass $m$.
} 
odology described in Ref. [18]. ${ }^{2}$ Initial boxes contain 256 particles in the gas phase and 500 particles in the solid slab. A minimum of $10^{6} \mathrm{MC}$ cycles are performed for equilibration and production. To characterize the phase behavior in the regime where microphase separation occurs, two identical boxes with a minimum of 256 particles on a lattice are used for initial configurations. At these lower temperatures, the GEMC method serves as an efficient phase space sampling algorithm akin in spirit to the grand canonical MC and parallel-tempering $\mathrm{MC}$ used in previous studies of systems with competing attractive and repulsive interactions [13]. It should be noted, however, that the approach implemented here is more efficient at detecting complex phase boundaries. In particular, upon crossing from a gas-solid coexistence region to a microphase separated region, the parallel-tempering MC sampling efficiency is dramatically reduced.

Before discussing the impact on phase equilibrium of the repulsive portion of the potential, we make some brief comments on the purely attractive case in two dimensions. The phase diagrams of short-ranged attractive two-dimensional systems have not been investigated as thoroughly as their three-dimensional counterparts. The existence of highdensity crystal-crystal coexistence in both two and three dimensions supports the notion that systems characterized by identical interactions in two and three dimensions are qualitatively similar [22]. While crystal-crystal coexistence does not occur in the temperature and density range of interest here, this phenomena is related to the metastability of the gas-liquid critical point, which does occur at densities and temperatures of concern in this work. With no repulsion $(A$ $=0$ ), we thus expect the liquid-gas coexistence to become metastable for $n \gtrsim 12$, so for $n=50-100$ the gas-liquid binodal is metastable and buried below the gas-solid coexistence line [23]. In three dimensions it is known that nucleation dynamics are dramatically influenced by this buried gas-liquid binodal [24-26]. Similar effects also appear in two dimensions, although metastable states are harder to observe, due to much faster nucleation. In the two-dimensional attractive case $(n=100, A=0)$, our visual inspection of the relaxation dynamics accompanying apparent spinodal decomposition gives $T_{c}=0.18 \pm 0.01$. This can be favorably checked by combining the arguments developed by Noro and Frenkel regarding corresponding states for short-ranged potentials [21] with the location of the critical point coordinates extracted from the results of Seaton and Glandt for the Baxter limit of adhesive disks [27], which further give an estimate of the critical density $\rho_{c}=0.6 \pm 0.1$. For the same potential form $(n=100, A=0)$, but in three dimensions, $T_{c}$ $=0.235 \pm 0.005[15]$ and $\rho_{c} \sim 0.5[18,28]$, while the threedimensional system studied here $(n=50, A=0)$ has $T_{c}$

\footnotetext{
${ }^{2}$ Local and nonlocal moves are equally frequent at low densities and high temperatures, and for gas-liquid equilibria. Otherwise, nonlocal moves comprise up to $90 \%$ of the total. Local moves are particle displacements $(94 \%)$, cluster displacements $(0.5 \%)$, rotations $(0.5 \%)$, and cleaving [31] (up to $1 \%$ ), as well as symmetric $(3 \%)$ and asymmetric ( 1 to $2 \%$ ) volume exchanges. Nonlocal moves are particle exchanges $(80 \%)$ and one- or two-box (10\% each) aggregation volume bias [32].
}
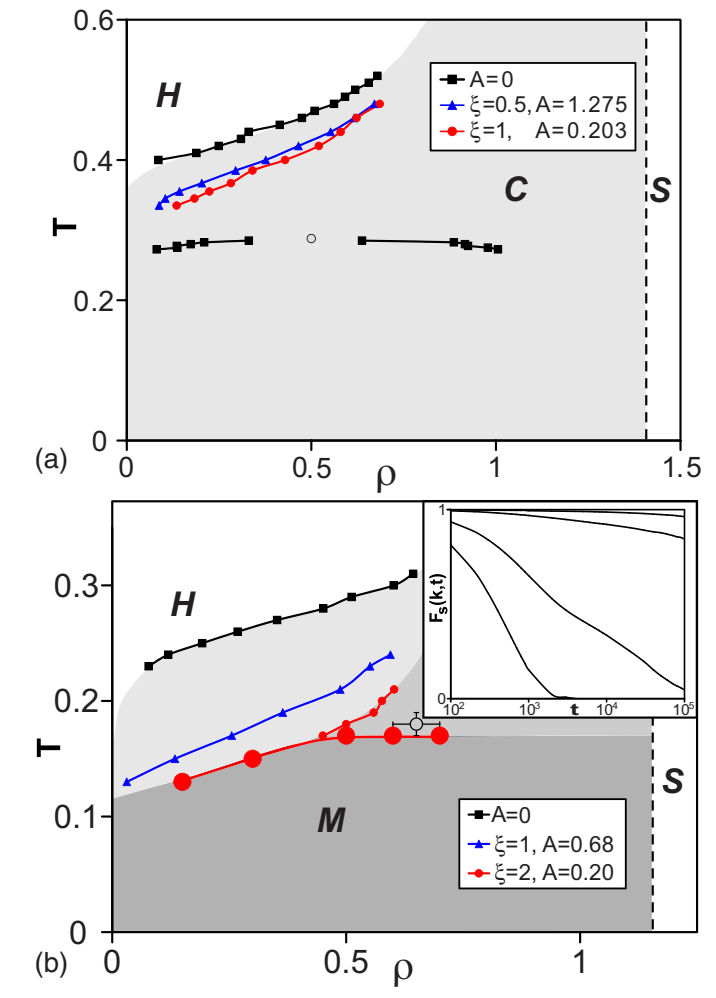

FIG. 1. (Color online) Phase diagrams for short-ranged attraction in (a) three $(n=50)$ and (b) two $(n=100)$ dimensions with increasing repulsion set by $A$ and $\xi$. The dashed line is the crystal close-packed density. Critical points (open circles) for purely attractive systems $(A=0)$ are obtained as described in the text. Small symbols indicate gas-solid and gas-liquid coexistence and large symbols the microphase boundary for $\xi=2$ and $A=0.2$. Uncertainty is smaller than the symbols, unless otherwise indicated. The phases are labeled M (microphase), H (homogeneous fluid), C (gas-crystal coexistence), and $\mathrm{S}$ (solid). Lines and shaded areas are guides for the eye. Inset: $F_{s}(k, t)=\left\langle\exp \left\{\mathbf{k} \cdot\left[\mathbf{r}_{i}(t)-\mathbf{r}_{i}(0)\right]\right\}\right\rangle$ from MD of the microphase forming system at the low- $k$ peak of the structure factor $k \sigma \approx 0.9$ for $\rho=0.6$ and $T=0.22,0.17,0.12$, and 0.08 , from left to right. For the higher two temperatures, trajectories are run for many times the relaxation time. For the lower two temperatures the system shows aging.

$=0.288 \pm 0.005[18]$. The metastable critical points are indicated in Fig. 1.

We now turn to a discussion of the impact of repulsion on phase behavior. In three-dimensional systems with attractive ranges similar to that of the $n=6$ (Lennard-Jones) potential, the addition of small amplitude long-ranged repulsion is known to slightly depress the gas-liquid spinodal and binodal lines [29]. Repulsion of greater amplitude or longer range transforms the depressed gas-liquid coexistence line into a microphase boundary where low- and high-density regions locally coexist, as in Fig. 1(b). Besides, repulsion destabilizes the crystal phase and thereby extends the stability of the homogeneous fluid phase to lower temperatures, as seen in Fig. 1. Where microphase separation occurs, this depresses the low-density microphase boundary. In this case the highdensity phase is crystalline, since the microphase boundary is below the gas-solid coexistence line. GEMC temperature scans allow us to visually pinpoint the boundary of the mi- 

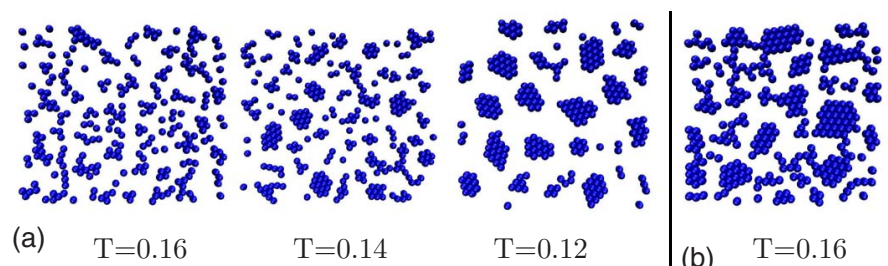

$\mathrm{T}=0.14$

$\mathrm{T}=0.12$

(b) $\mathrm{T}=0.16$
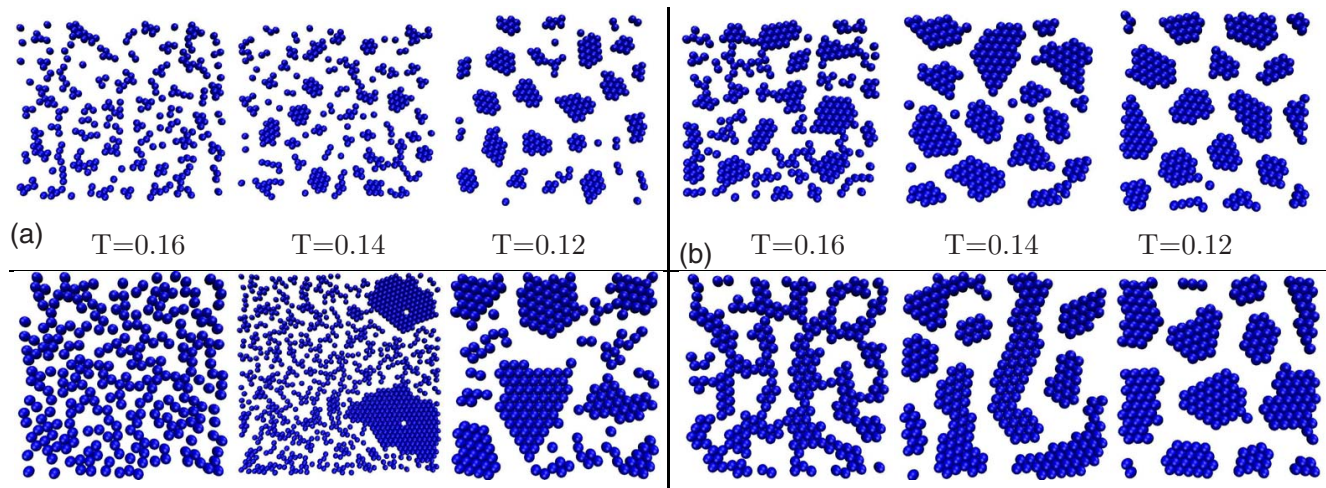

$\mathrm{T}=0.12$

(c) $\mathrm{T}=0.22$

$\mathrm{T}=0.18$

$\mathrm{T}=0.16$

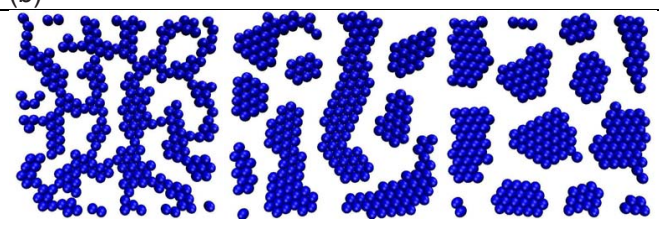

(d) $t=1.8 \times 10^{4} \quad t=2.05 \times 10^{6} \quad t=\infty$

FIG. 2. (Color online) Configurations ( $\xi=2, A=0.2$ ) equilibrated with GEMC simulations at (a) $\rho=0.3$, (b) $\rho=0.5$, and (c) $\rho=0.6$ as well as (d) $\rho=0.6 \mathrm{MD}$ evolution after quench to $T=0.12$ (first two panels) and GEMC thermodynamic outcome (third panel).

crophase region. General arguments indicate that fluctuations alter the nature of the critical point, rendering the transition weakly first order [30].

At this stage it is useful to directly observe representative equilibrium configurations obtained during GEMC runs, as in Figs. 2(a)-2(c). Configurations below the microphase transition show slightly irregular domains, due to finitetemperature entropic fluctuations. For this system at the densities and temperatures shown, compact cluster states are more stable than extended (lamellar) states, which were not observed. The second and third panels of Fig. 2(c) illustrating configurations just above and below the microphase line are particularly illuminating. The dynamical behavior upon a rapid quench below the microphase separation line is shown in the first two panels of Fig. 2(d). ${ }^{3}$ The evolution of the system from the second to the third panel of Fig. 2(d) is extremely slow via conventional MD. The dramatic increase in time scales upon cooling the system below the microphase separation can be seen from the self-intermediate scattering function $F_{s}(k, t)$ in the inset of Fig. 1(b). Fluctuations on the order of the characteristic domain length scale decay so slowly that the structure is effectively dynamically arrested.

The dynamical behavior shown in Fig. 2(d) is clearly relevant for the mechanism of gelation of the system. In particular, consider a slightly deeper quench at a lower density than that shown in Fig. 2(d). In Fig. 3(a) a quench of the system is made at $\rho=0.5$ to $T=0.08$. After rapid initial transient dynamics, where a tenuous percolating structure is formed, the dynamics becomes anomalously slow. This system may be characterized as a gel. Due to the depth of the quench, we did not observe substantial structural coarsening [as is observed in Fig. 2(d)] over the duration of the simula-

${ }^{3} \mathrm{MD}$ integration with step $\Delta t=0.001$ was performed for system sizes $N \geqslant 256$. Cooling is done from a fluid configuration to the set temperature by resampling velocities every $t_{\text {cool }}=10$. The type of dynamics does not influence the qualitative results strongly [5], so other protocols are not studied. Slower cooling rates allow the system to reach configurations closer to equilibrium [15]. The potential is truncated at $r_{c}=5(3.3)$ for $\xi=2(1)$, which alters the microphase patterning [13], but here the results do not depend on the form of the ground state, since GEMC and MD runs use equal $r_{c}$. tions, although the energy of the system is slowly evolving in time. The similar evolution is expected to be much slower in the case of a polydisperse, three-dimensional system. For comparison, the equilibrium state of the system (as found via GEMC simulation) is also shown in Fig. 3. Note that the structure of the gel does not resemble the equilibrium state in any regard, despite the fact that the energy per particle in the gel is quite close to that of particles in their cluster ground state. In addition, we find via the GEMC method no evidence of extended microphase structures at the density and temperature of Fig. 3. Regardless, the short-time critical-like fluctuations that exist during the initial stages of microphase separation are sufficient to generate configurations that span space and evolve anomalously slowly, due to strong, shortranged bonding between particles.

The results presented above are clearly of importance for understanding the general routes to gelation in colloidal systems with competing short-ranged attraction and long-ranged repulsion. We find, rather robustly, that gels may form via arrested microphase separation. This is analogous to the purely attractive case, where gels are formed by arrest of global phase separation. This picture is in contrast to that of Refs. $[15,16]$, where gelation is a consequence of nearequilibrium vitrification of clusters that are stabilized by the repulsive interactions. We have explicitly demonstrated here that, as in the purely attractive case, the gel may be stabilized by the attraction between particles. More interestingly, the gel structure bears no similarity to the clusters that exist in

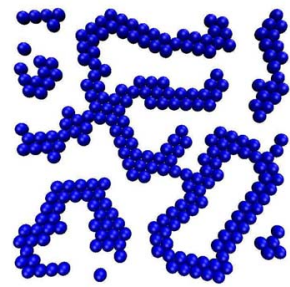

(a)

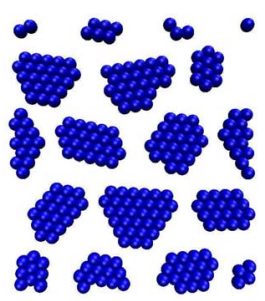

(b)
FIG. 3. (Color online) For $\rho=0.5(\xi=2, A=0.2)$ (a) nearly arrested gel configuration obtained by $\mathrm{MD}$ at $t=5 \times 10^{5}$ after a quench to $T=0.08$ and (b) an equilibrium configuration at $T=0.08$ found by GEMC simulation. 
thermodynamic equilibrium. We find that in the system studied here, the only role of the repulsion is to select the symmetry of the ground state(s). Our results are fully consistent with, but more general than, the suggestion that disordered configurations of anisotropic microphase structures are connected to gelation $[11,14,17]$. Indeed, we have directly demonstrated that extended microphase structures are not involved during the formation of the gel in Fig. 3. Furthermore, preliminary studies in three dimensions for the system studied in Ref. [15] show that for rapid, deep quenches gels may be formed in the same manner. This leads us to believe that both the routes presented here and in Refs. $[15,16]$ are possible, and will likely depend intimately on the details of the quench protocol. In general, lamellar and tubular structures (as well as other microphase textures) may comprise the building blocks of the disordered weak solids found in colloidal systems with depletion attraction and long-ranged charge repulsion, but our results demonstrate that they need not. The disordered lamellar and tubular examples are simply some of the many ways in which microphase separation may be arrested in a manner directly analogous to the arrest of global phase separation. The collection of all such ways perhaps forms the simplest generic route to the gel state at intermediate volume fractions in attractive systems with charge frustration. The search for more exotic routes, which may be operative at very low volume fractions and where charge repulsion plays a more active role, may be greatly facilitated by the use of the GEMC approach as outlined here.

This work was supported by Grants No. NSF-0134969 and No. FQRNT-91389 (P.C.). We thank E. Del Gado, D. Frenkel, P. Lu, and K. Miyazaki for discussions and comments.
[1] M. E. Cates M. Fuchs, K. Kroy, W. C. K. Poon, and A. M. Puertas, J. Phys.: Condens. Matter 16, S4861 (2004).

[2] N. A. M. Verhaegh, D. Asnaghi, H. N. W. Lekkerkerker, M. Giglio, and L. Cipelletti, Physica A 242, 104 (1997).

[3] S. Manley, H. M. Wyss, K. Miyazaki, J. C. Conrad, V. Trappe, L. J. Kaufman, D. R. Reichman, and D. A. Weitz, Phys. Rev. Lett. 95, 238302 (2005).

[4] G. Foffi, C. De Michele, F. Sciortino, and P. Tartaglia, Phys. Rev. Lett. 94, 078301 (2005).

[5] G. Foffi, C. De Michele, F. Sciortino, and P. Tartaglia, J. Chem. Phys. 122, 224903 (2005).

[6] E. Zaccarelli, S. V. Buldyrev, E. La Nave, A. J. Moreno, I. Saika-Voivod, F. Sciortino, and P. Tartaglia, Phys. Rev. Lett. 94, 218301 (2005).

[7] E. Del Gado and W. Kob, Europhys. Lett. 72, 1032 (2005).

[8] S. A. Shah, Y. L. Chen, S. Ramakrishnan, K. S. Schweizer, and C. F. Zukoski, J. Phys.: Condens. Matter 15, 4751 (2003).

[9] P. N. Segre, V. Prasad, A. B. Schofield, and D. A. Weitz, Phys. Rev. Lett. 86, 6042 (2001).

[10] S. Mossa, F. Sciortino, P. Tartaglia, and E. Zaccarelli, Langmuir 20, 10756 (2004).

[11] F. Sciortino P. Tartaglia, and E. Zaccarelli, J. Phys. Chem. B 109, 21942 (2005).

[12] R. P. Sear, S. W. Chung, G. Markovich, W. H. Gelbart, and J. R. Heath, Phys. Rev. E 59, R6255 (1999).

[13] A. Imperio and L. Reatto, J. Phys.: Condens. Matter 16, S3769 (2004)

[14] A. de Candia, E. Del Gado, A. Fierro, N. Sator, M. Tarzia, and A. Coniglio, Phys. Rev. E 74, 010403(R) (2006).

[15] F. Sciortino, S. Mossa, E. Zaccarelli, and P. Tartaglia, Phys.
Rev. Lett. 93, 055701 (2004).

[16] J. Wu, Y. Liu, W. R. Chen, J. Cao, and S. H. Chen, Phys. Rev. E 70, 050401(R) (2004).

[17] M. Tarzia and A. Coniglio, Phys. Rev. Lett. 96, 075702 (2006).

[18] P. Charbonneau and D. R. Reichman, Phys. Rev. E 75, 011507 (2007).

[19] H. Liu, S. Garde, and S. Kumar, J. Chem. Phys. 123, 174505 (2005).

[20] A. Z. Panagiotopoulos, Mol. Simul. 9, 1 (1992).

[21] M. G. Noro and D. Frenkel, J. Chem. Phys. 113, 2941 (2000).

[22] D. Frenkel, P. Bladon, P. Bolhuis, and M. Hagen, Physica B 228, 33 (1996).

[23] M. Hasegawa and K. Ohno, J. Phys.: Condens. Matter 9, 3361 (1997).

[24] P. R. ten Wolde and D. Frenkel, Science 277, 1975 (1997).

[25] A. P. Gast, C. K. Hall, and W. B. Russel, J. Colloid Interface Sci. 96, 251 (1983).

[26] M. H. J. Hagen and D. Frenkel, J. Chem. Phys. 101, 4093 (1994).

[27] N. A. Seaton and E. D. Glandt, J. Chem. Phys. 84, 4595 (1986).

[28] M. A. Miller and D. Frenkel, J. Chem. Phys. 121, 535 (2004).

[29] D. Pini, J. L. Ge, A. Parola, and L. Reatto, Chem. Phys. Lett. 327, 209 (2000).

[30] S. A. Brazovskii, Sov. Phys. JETP 41, 85 (1975).

[31] S. Whitelam and P. L. Geissler, e-print arXiv:cond-mat/ 0508100 (unpublished).

[32] B. Chen and J. I. Siepmann, J. Phys. Chem. B 104, 8725 (2000). 